\title{
FORMULATION AND EVALUATION OF SOLID SELF MICRO EMULSIFYING DISPERSIBLE TABLET OF PIROXICAM
}

\author{
GIRISH S. H. ${ }^{1}$, SURABHI C. ${ }^{1}$, SATHISH BABU P. ${ }^{1}$, MEENAKSHI S. ${ }^{2}$, SIDDARAMAIAH ${ }^{3}$, NITHIN K. S. ${ }^{4}$, D. V. GOWDA ${ }^{1 *}$
}

${ }^{1 *}$ Department of Pharmaceutics, JSS College of Pharmacy, JSS Academy of Higher Education and Research, Mysuru 570015, Karnataka, India, ${ }^{2}$ Department of Prosthodontics, JSS Dental College and Hospital, JSS Academy of Higher Education and Research, Mysuru 570015, Karnataka, India, ${ }^{3}$ Department of Polymer Science and Technology, S J College of Engineering, JSS Science and Technology University, Mysuru 570006, Karnataka, India, ${ }^{4}$ Department of Chemistry, The National Institute of Technology, Mysuru 570008, India Email: girish.jssuni@gmail.com

Received: 13 Nov 2020, Revised and Accepted: 06 Jan 2021

ABSTRACT

Objective: The aim of this study was to formulate the solid self-micro emulsifying dispersible tablets for promoting the dissolution of Piroxicam.

Methods: Solubility study test was performed to know the solubility of various oil phase, surfactants, cosurfactants. Self-emulsifying grading test was done by visual grading system. Ternary phase diagrams and droplet size analysis test were performed to screen and optimize the Piroxicamself microemulsifying drug delivery system (SMEDS). Then microcrystalline cellulose (KG802) was added as a suitable adsorbent and dispersible tablet were prepared by wet granulation compression method.

Results: The final composition of Piroxicam-SMEDS was oil phase (oleic acid, 23\%), surfactant (Cremophor R H-40,61\%), co-surfactant (PEG$400,16 \%$ ) based on the result of solubility test, self-emulsifying grading test, droplet size analysis and ternary phase diagrams. Microcrystalline cellulose (KG802) was selected based on dissolution study (98.35\%) and added to liquid Piroxicam-Smeds formulation to form dispersible tablets. The in vitro dissolution study showed $98.02 \%$ of drug release from Piroxicam-SMEDS tablets.

Conclusion: Piroxicam-Self microemulsifying dispersible tablets have increased the solubility and bioavailability of the Piroxicam to a greater extent. SMEDS formulation can help the solubility of poorly water-soluble drugs.

Keywords: Proxicam, solid self microemulsifying system, Dispersible tablet

(C) 2021 The Authors. Published by Innovare Academic Sciences Pvt Ltd. This is an open access article under the CC BY license (https://creativecommons.org/licenses/by/4.0/) DOI: https://dx.doi.org/10.22159/ijap.2021v13i2.40238. Journal homepage: https://innovareacademics.in/journals/index.php/ijap

\section{INTRODUCTION}

The oral route of administration is most evident and well-fancied by every patient [1], which is economical and simplest route of administration and does not require any devices, which is helpful for self-medication [2]. In and around if we look $40 \%$ of new medication have poor solubility in water and ultimately results in poor oral bioavailability, which is the key challenge to formulate an oral dosage form $[3,4]$. Since dissolution is the rate-limiting step, remodeling in the formulation is the correct approach to reinforce absorption and ultimately bioavailability of those medications $[5,6]$. Several formulation approaches like complexation [7], solid dispersions [8], and nanosuspensions [9] are used to unravel this drawback; however, these approaches have some limitations like less stability and heavy exorbitant methods.

According to Biopharmaceutical arrangement, Piroxicam (PX) is a non-steroidal anti-inflammatory drug (NSAIDs) which was classified under the category of Class II drug (low solubility and high porosity)[10]. Generally, the NSAIDs with an extended half-life and it is employed to treat pain related to rheumatism, degenerative arthritis, arthritis, and numerous contractile organ disorders. NSAIDs block enzyme, which ultimately results in inflammation, pain, and fever is reduced. Balakrishna et al. [11] suggested that the self-micro emulsifying drug delivery system (SMEDS) is the effective pharmaceuticaltechnologyto beat issues of drugs with low solubility and poor oral absorption. SMEDS contains isotropic mixtures of natural or artificial oils, surfactants, and co-surfactants. This framework not just improves sedate solubilization but also improves the deliverance and absorption features, as it then contains the dissolve style of the drug within the formulation; therefore, tiny droplet size giving an increased cover area. These systems effectively emulsify once exposed to channel fluids to make oil in water microemulsion with nanometric droplet size. Self-microemulsifying drug delivery system (SMEDS) enhances in vitro dissolution and improves the in vivo absorption of lipotropic medication [12, 13]. SMEDS is the system with the droplet size starting from 20 to $200 \mathrm{~nm}[14,15]$. The solubilization capability was to research SMEDS for the nonsteroidal anti-inflammatory drug. The small-sized droplets of SMEDS supply a rise in the dissolution rate and bioavailability of medicine $[16,17]$.

Still, SMEDSs, which are fluid formulation, have some disadvantages, that some varieties of dosage forms are available, which have under the stability and low portability throughout the manufacturing method in which interaction occurs to filling as well as capsule shell. Hence surmount with these difficulties, the lipid formulation can be converted into a simple solid dosage form with the help of a proper adsorbent. Various types of hydrophilic as well as hydrophobic solid adsorbent could be used like Magnesium trisilicate, colloid silica, bentonite, dextran, etc have reached absorbing the liquid SMEDS and different techniques (e. g., extrusion-spheronization, spray drying, and granulation, etc.) [18], are utilized to serve into pellets, powders or different solid dosage form can be additionally filled into a capsule or can be packed into the tablet [19]. Among these ways, surface assimilation to solid carriers could be an easy and straight forward technique, giving a stable free-flowing S-SMEDS [20]. Density porous carriers with massive areas i.e. microcrystalline cellulose KG802 were employed to boost the dissolution rate of medicine like a beta-blocker [21]. Hence, the present study was focused to develop and evaluate Piroxicam loaded SMEDS using different oil and surfactant mixtures.

\section{MATERIALS AND METHODS}

\section{Materials}

Piroxicam (99.2\%) was received as a gift sample from Micro labs Pvt Ltd (Banglore, India). Castor oil, olive oil, corn oil was purchased from Alaba Analytical Laboratories (Surat, India). Oleic acid was purchased from Pas and Speciality Chemicals (Rajkot, India). Tween 80 was purchased from Loba chemicals (Mumbai, India). Cremophor RH-40 was purchased from Zeel Pharmaceuticals (Mumbai, India), 
OP-10 was purchased from K. K. Chempro India Private Ltd (Mumbai, India). Ethanol, butanol, isopropyl alcohol was purchased from Loba chemicals (Mumbai, India). PEG-400 was purchased from Montage Chemicals Pvt Ltd (Mumbai, India). Glycerol was purchased from Siddi Vinayak Industries (Ahmedabad, India).

\section{Methods}

\section{Drug excipient compatibilities}

\section{The fourier-transform infrared spectroscopy (FTIR)}

The spectra of the drug, excipients, SMEDS, and selfmicroemulsifying mouth dissolving film formulation formed were noted and recorded. Briefly, a solid sample was packed together with regarding $100 \mathrm{mg}$ dried potassium bromide $(\mathrm{KBr})$ into a disc. For liquid specimens, few drops of the sample were dripped over the $\mathrm{NaCl}$ aperture plate and sandwiched it beneath the aperture plate, and made sure that there were no gas bubbles were at bay [22]. The sample endorsed the formation of a skinnyliquid membrane between the 2 aperture plates. Thereafter, the sample was scanned for absorbance over the vary from 4000 to $400\left(\mathrm{~cm}^{-1}\right)$ wavenumbers. The obtained spectrum was then matched with the normal cluster frequencies of PX.

\section{Selection of the self-microemulsified drug delivery system component}

\section{Solubility study of the PX}

PX dissolvability was known in oil, surfactant, and co-surfactant by the shake-flask technique. Castor oil, olive oil, oleic acid, corn oil were used as an oil, Tween 80, Cremophor R H-40, OP-10 were used as Surfactant and PEG-400, ethanol, glycol, Isopropyl alcohol, glycol, butanol, solutol were used as Co-surfactants. It was done by solubilizing an abundance amount of medication in $2 \mathrm{ml}$ of the medium. The blend was then vortexed first and kept for $72 \mathrm{~h}$ at $25^{\circ}$ in an orbital shaking hatchery (Dolphin) for solubilization.

After harmony, it was centrifuged in $10,000 \mathrm{rpm}$ for $15 \mathrm{~min}$. The supernatant fluid was diluted with $0.1 \mathrm{M}$ methanolic $\mathrm{HCl}$ and medication were evaluated at $334 \mathrm{~nm}$ [23] by utilizing a UV spectrophotometer by placing a blank. The blank was then formulated by mixing oil, surfactant, and co-surfactant in $0.1 \mathrm{M} \mathrm{HCl}$ in methanol with the same dilution concerning the example [24].

\section{Self-emulsifying assess test}

According to Yang et al., elective oil (Castor oil, olive oil, oleic acid, corn oil), surfactants (Tween 80, Cremophor R H-40, OP-10), and cosurfactants (Butanol, ethanol, solutol, glycerol, PEG 400, Isopropyl glycol) were homogenized pertaining to the result obtained during solubility obtained by $3: 4: 3(\mathrm{w} / \mathrm{w} / \mathrm{w})$ in cells by vortexing for about $5 \mathrm{~min}$ [25]. Next, place $1 \mathrm{ml}$ of mixture into the $100 \mathrm{ml}$ of water (37 ${ }^{\circ} \mathrm{C}$ ) using magnetic stirring to witness the emulsion development method and ultimate appearance. The event was classified into 5 classes by a visual grading methods according to Anna et al. as first grade which expressed the accelerated emulsification formed in 1 min and making a clear or light blue formation of microemulsion, second grade, which expressed the accelerated emulsification formed in $1 \mathrm{~min}$ and making a few clear and light whitish-blue emulsion, third grade which expressed the emulsification delayed within 2 min and making a bright white liquid, fourth grade which expressed the emulsification more delayed than of 2 min with dark dusky and lightly oil form, fifth grade which expressed neither the emulsification problems nor the presence of a large number of oil droplets [26].

\section{Optimizing the formulated SMEDS}

The single-factor approaches were taken for optimizing the SMEDS. The required proportion of the oil, co-surfactant, and surfactant in liquid SMEDS were prepared by using droplet size (DS) and the Polydispersity-index (PDI) formed by microemulsion. The Microemulsion having a tiny droplet size of around 10 to $100 \mathrm{~nm}$ can give an immense surface region that hinders the Para-glycoprotein interfered drug efflux and first-pass stomach metabolism, which also increases the GIT layer permeability. Prime, charge proportion of cosurfactant and the surfactant were fixed to $3: 1$ and the mass percentage of oil was extended from 10 percent to 50 percent. PDI and DS formed of microemulsions were decided by employing a Zetasizer (Malvern Instruments, Ltd., UK). The weight percentage formed by the co-surfactant and surfactant was prolonged from $5 \%$ to $25 \%$ and $30 \%$ to $70 \%$, and a weight percentage of oil was fixed at $25 \%$.

\section{Development of the liquid SMEDS}

PX (100 milligrams) were suspended to one $\mathrm{ml}$ of a blend carrying $23 \%$ Oleic acid, $61 \%$ Cremophor-RH 40 , and 16\% PEG 400. The terminal mixture produced were then vortexed till the plain admix is achieved. The ultimate drug present in liquid SMEDS was 10\% $(\mathrm{w} / \mathrm{w})$. The polydispersity index and particle sizes of the emulsion produced were estimated by Zetasizer.

\section{Preparation of PX-SMEDS dispersible tablet}

\section{Absorbing substances}

Based on the adsorption potential among various substances, including the percentage of PX discharged from the medium by 45 min, the exact absorbing substances were calculated to make the Solid-SMEDS.

\section{Development of PX-SMEDS dispersible doses}

Diluent like MCC KG 802, disintegrating agent (PVPP-XL), lubricants such as aerosil were combined to the PX-SMEDS to develop PX micro emulsifying dispersible tablet by using the wet granulation and compression technology. Afterward in vitro dissolution were carried out to the PX compact self-microemulsifying dispersible tablet were examined.

\section{In vitro dissolution profile study}

The in vitro dissolution of PX compact self-micro emulsifying dispersible tablets comprising $5 \mathrm{mg}$ PX were conducted in the dissolution apparatus. Dissolution requirements were purified water, $0.1 \mathrm{M}$ HCL solution with $\mathrm{pH} 6.7$ phosphate buffer solution, $37 \pm 0.5{ }^{\circ} \mathrm{C}, 50 \mathrm{rpm}, 250 \mathrm{ml} 4 \mathrm{ml}$ of the medium was withdrawn at 1 , $5,10,20,30,45,60$, and 120 min by replacing an equivalent volume of a similar temperature blank.

The switched samples were drained by using a membrane filter of $0.45 \mathrm{~mm}$, then the filtrate obtained was weakened by methanol, and then by using HPLC the concentration of the PX present in diluent was assayed. When correlated with tablets, the dissolution profile obtained from free PX was further made in a similar dissolution condition.

\section{RESULTS AND DISCUSSION}

\section{Drug excipient compatibilities study}

Fig. 1 noted the FTIR spectra of PX. According to Bunaciu et al., PX has two interconvertible crystalline forms, i. e the needle and cubic forms The IR $1629.90 \mathrm{~cm}-1$ was accredited to the stretching of amide carbonyl groups of a cubic form, implying that the cubic form of PX was used. The main characteristic peak of PX was $3338 \mathrm{~cm}-1$ for secondary amine $\mathrm{N}-\mathrm{H}$ stretch, 3446.91 $\mathrm{cm}-1$ for $\mathrm{O}-\mathrm{H}$ stretching, and $1182.40 \mathrm{~cm}-1$ for $\mathrm{S}=0$ asymmetric stretching [27]. SMEDS formulation presented peaks at 1760.70 $\mathrm{cm}-1$ for $\mathrm{C}=0$ amide carbonyl group, $3446.91 \mathrm{~cm}-1$ for $\mathrm{N}-\mathrm{H}$ stretch, 3628.22 for $\mathrm{O}-\mathrm{H}$ stretching, and 1215.19 for $\mathrm{S}=0$ asymmetric stretch.

The N-H group of the amide in PX formed the hydrogen bond with a carboxyl group of SMEDS excipients (dissolvable and dilute form for PX) and this decreased electron density of nitrogen. This advances to the shifting of the $\mathrm{C}=\mathrm{O}$ stretch of amide carbonyl. The IR spectra of formulation (SMEDS with drug) show the absence of exceptional changes in the position of the individual infrared absorption bands of the drug in formulation, proposing that there is no interaction between PX and excipients. 


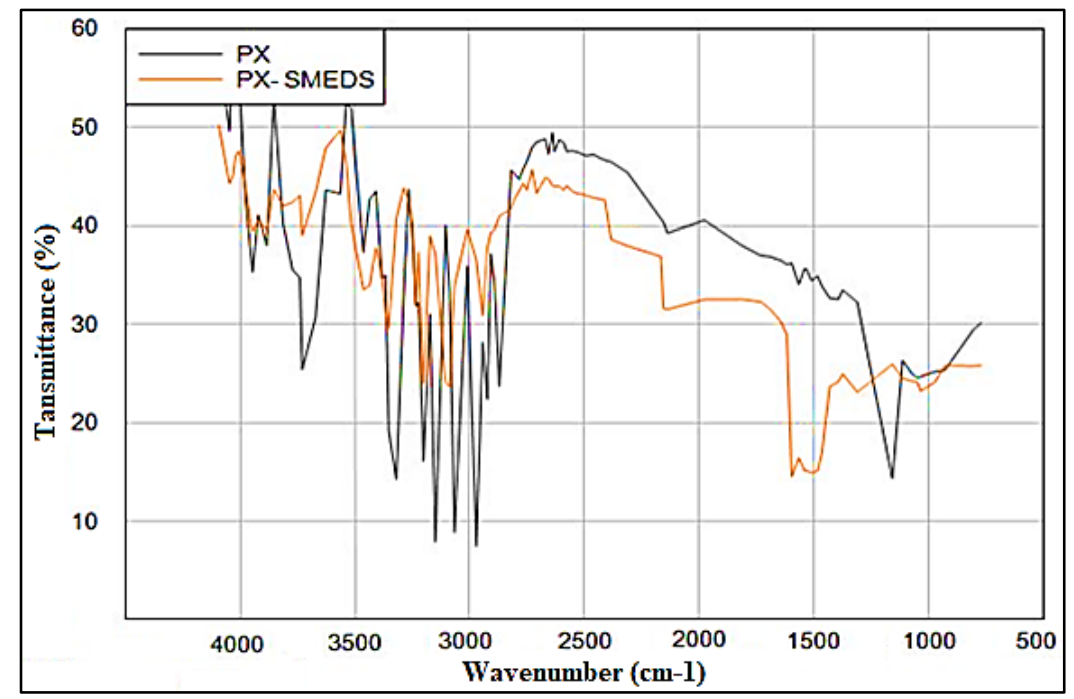

Fig. 1: FTIR spectra of pure PX and SMEDS accompanied with PX

\section{The solubility of PX}

Results of PX solubility in various oils have been displayed in the figure. It was recognized that oleic acid having a higher concentration of esters [28], the product illustrated greater solubility in oleic acid and hence it has been chosen as an oil phase. It is known that nonionic surfactants are stronger than ionic surfactants [29]. Reversible changes in intestinal mucosal permeability are expected to occur [30]. Those with HLB within 4.3 and 16 have been tested. The final surfactant was chosen by its capacity to emulsify and deliver the transmittance. Cremophor-RH 40 increased transmittance percentage, indicating a good ability to emulsify as displayed in fig. 2. In these surfactants, PX displayed better solubility in Cremophor RH 40 and was used for further testing. Co-surfactants were made to be screened for promoting the emulsification ability of the selected surfactant. The percentage of the transmittance and emulsification time were carried out using PEG 400 amongst all the co-surfactants screened, PEG 400 showed the highest percent transmittance when used along with Cremophor RH 40. Cremophor RH 40 having higher HLB i.e. 15. According to Zeng et al. more HLB values of surfactant lead to increase active dispersion and more precise emulsion droplet size on an extension to the aqueous phase [31]. Hence PEG 400 was selected as Co surfactant.

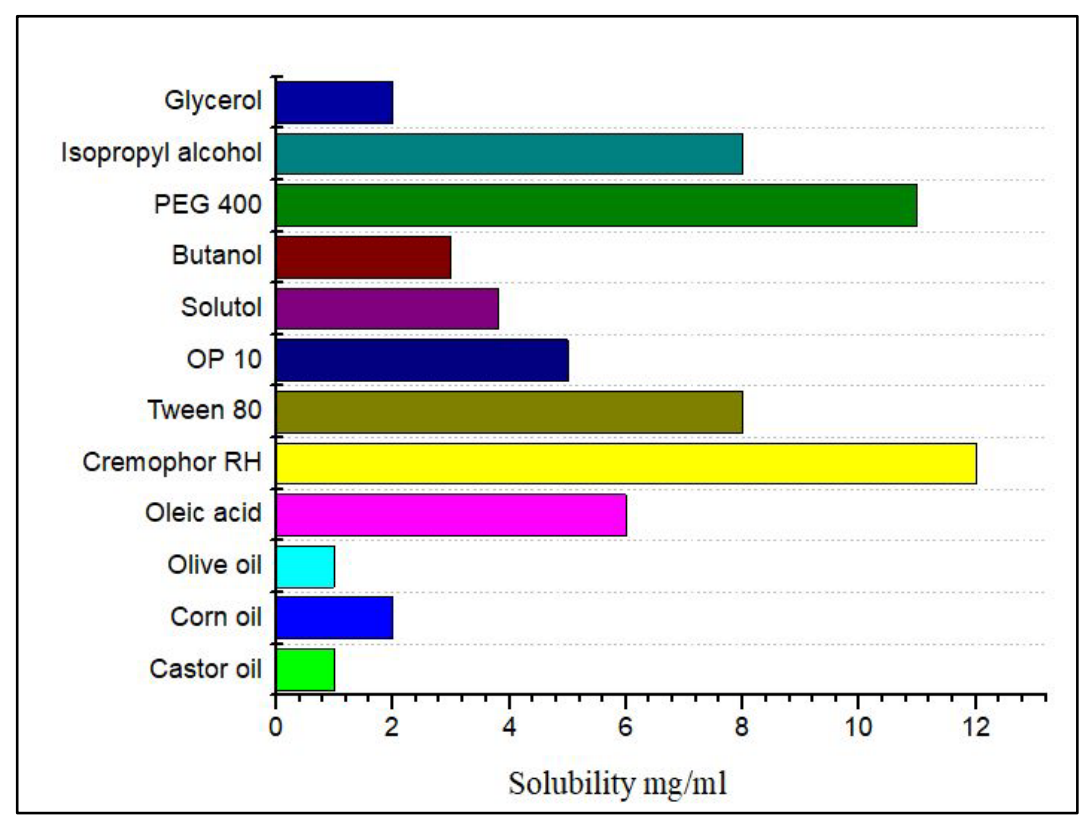

Fig. 2: The solubility studies of PX in various oil, Surfactant, Co-surfactant

\section{Self-emulsifying assesses test}

Visible grading effects concerning microemulsions offered over various SMEDS compounds have been given in table 1, having PEG 400 set as co-surfactant. Later, surfactants like Cremophor RH 40, OP-10, and Tween 80 were combined amidst all oils. The marked surfactants, which revealed the poor capacity to form a microemulsion, were graded as D and E and which showed the good capacity of forming microemulsion were graded A and B [32]. However, Cremophor R H-40 produced fine microemulsion among all, as most of it got visual class as B. Hence Cremophor R H 40 was chosen as a surfactant in the study, whereas castor oil, olive oil, oleic acid, and corn oil were approximately elected as an oil phase for the further selection procedure. 
Table 1: Visual grading effects obtained by a compatability study having a ratio of surfactant, oil, and co-surfactant fixed at 4:3:3(w/w/w)

\begin{tabular}{lllll}
\hline S. No. & Oil & Cremophor RH-40 & OP-10 & Tween80 \\
\hline 1 & Oleicacid & B & B & D \\
2 & Castoroil & B & E & E \\
3 & Oliveoil & B & D & D \\
4 & Cornoil & B & D & D \\
\hline
\end{tabular}

Note: Good capacity of forming microemulsion (A and B), Poor capacity of forming microemulsion (C,D and E)

\section{Preparation of ternary phase diagram}

Ternary phase charts concerning the oils (olive oil, castor oil, oleic acid, corn oil), surfactant ie, Cremophor RH-40, and co-surfactant PEG 400 were constructed without the presence of PX to recognize the self-emulsifying areas [33]. The highest microemulsion area was seen at oleic acid (fig. $3 \mathrm{C}$ ) which was selected as an oil phase. Hence the Oleic acid-Cremophor RH-40, PEG 400 lends was chosen as a liquid SMEDS for PX and they were chosen for additional study.

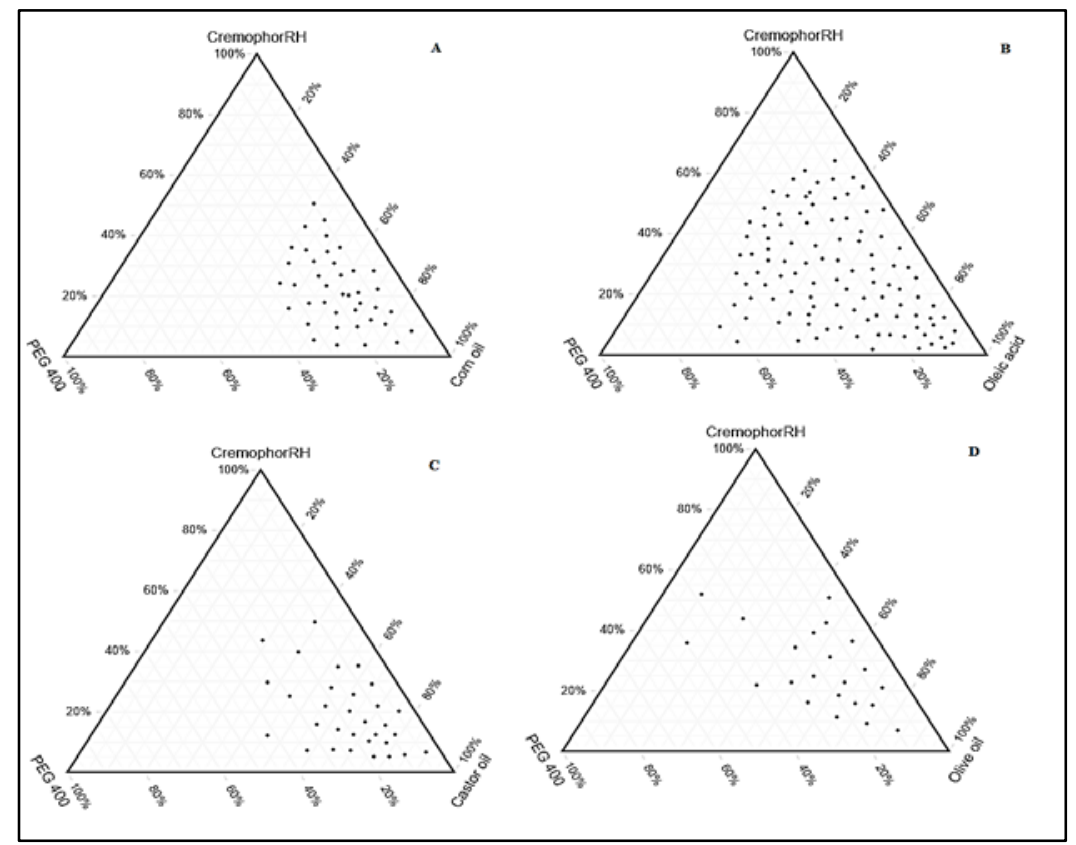

Fig. 3: Ternary phase diagram formed of variousoils, surfactant, Co-surfactant; A. cornoil, cremophor RH-40, PEG400; B. Oleicacid, Cremophor RH-40, PEG400; C. Castoroil, Cremophor RH-40, PEG400; D. Olive oil, Cremophor RH-40, PEG400

\section{Optimizing the formulated SMEDS}

The droplet area of the emulsion will be an influential factor during self-emulsification execution as it will define the degree and the extent of a drug discharge and absorption [34]. While increasing the percentage of the oil phase during the formulation (table 2), DS and
PDI will increase, while the increasing percentage of surfactant DS and PDI will be decreased. When the co-surfactant level is more than $15 \%$, both PDI and DS have progressed. As the Km increases, PDI and DS slowly decrease. Hence accepted optimized formulation for PX-SMEDS would be surfactant (Cremophor-RH 40) 60\%, oil phase (Oleic acid) 25\%, co-surfactant (PEG 400) 15\%.

Table 2: Influence of formulation on polydispersity index and particle size

\begin{tabular}{|c|c|c|c|c|}
\hline Oil & Surfactant & Co-surfactant & P. S \pm SD $^{*}(\mathrm{~nm})$ & PDI \pm SD* \\
\hline 10 & 67.5 & 22.5 & $8.92 \pm 0.82$ & $0.17 \pm 0.011$ \\
\hline 20 & 60 & 20 & $14.45 \pm 0.85$ & $0.23 \pm 0.025$ \\
\hline 25 & 56.25 & 18.75 & $16.69 \pm 0.96$ & $0.23 \pm 0.023$ \\
\hline 30 & 52.5 & 17.5 & $42.38 \pm 0.56$ & $0.44 \pm 0.015$ \\
\hline 40 & 45 & 15 & $126.36 \pm 1.01$ & $0.35 \pm 0.025$ \\
\hline 50 & 37.5 & 12.5 & $135.4 \pm 0.69$ & $0.68 \pm 0.089$ \\
\hline 25 & 30 & 45 & $82.6 \pm 0.98$ & $0.38 \pm 0.023$ \\
\hline 25 & 30 & 44 & $81.5 \pm 0.96$ & $0.35 \pm 0.011$ \\
\hline 25 & 50 & 25 & $47.5 \pm 0.36$ & $0.61 \pm 0.089$ \\
\hline 25 & 60 & 15 & $14.98 \pm 0.32$ & $0.13 \pm 0.056$ \\
\hline 25 & 70 & 05 & $12.01 \pm 0.69$ & $0.11 \pm 0.042$ \\
\hline 25 & 70 & 05 & $14.25 \pm 0.01$ & $0.22 \pm 0.054$ \\
\hline 25 & 65 & 10 & $13.23 \pm 0.75$ & $0.12 \pm 0.035$ \\
\hline 25 & 60 & 15 & $14.2 \pm 0.21$ & $0.19 \pm 0.053$ \\
\hline 25 & 55 & 20 & $24.3 \pm 0.36$ & $0.54 \pm 0.101$ \\
\hline 25 & 50 & 25 & $60.9 \pm 1.89$ & $0.65 \pm 0.069$ \\
\hline
\end{tabular}

Note: *SD: Standard Deviation, n=3, *P. S: Particle size, *PDI: Polydispersity Index. 


\section{Absorbing substances}

During the experiment, it was found that the absorption potential offered by insoluble substances were greater than that offered by soluble materials (fig. 4). Through our analysis silica had the greatest absorption potential; the percentage of PX released during $45 \mathrm{~min}$ was lesser to 79 percent, whereas the percentage of PX discharged from the MCC KG802 would exceed 98.35 $\pm 0.25 \%$ (table 3). The reception capability of the MCC KG 802 was $1 \mathrm{~g}$. Hence, MCC KG 802 was taken as solid material.

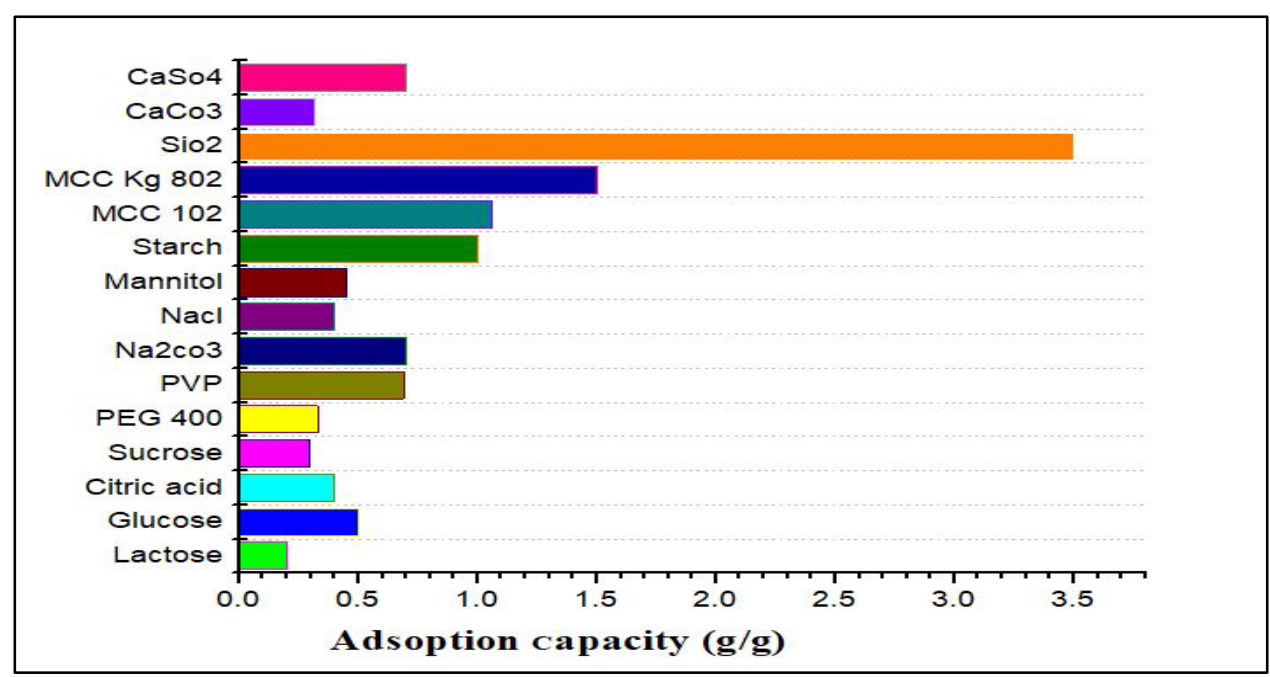

Fig. 4: The absorption potential obtained by 1 gram of soluble and insoluble materials $(\mathrm{mean} \pm \mathrm{SD} n=3)$

Table 3: The dissolution (\%) obtained by different types of solid materials during 45 min

\begin{tabular}{ll}
\hline Solid materials & $\mathbf{4 5}$ min dissolution (\%)* \\
\hline MCC101 & $83.44 \pm 5.45$ \\
MCCKG802 & $98.35 \pm 0.25$ \\
MCC102 & $80.37 \pm 3.54$ \\
Starch & $76.58 \pm 0.23$ \\
Sio 2 & $76.22 \pm 1.04$ \\
\hline
\end{tabular}

Note: $*$ Data represented as mean $\pm \mathrm{SD}(\mathrm{n}=3)$

\section{In vitro drug release study}

For L-SMEDS, in vitro dissolution studies showed around $81.23 \%$ drug release over 5 min and over $98.97 \%$ within $45 \mathrm{~min}$. For the S-SMEDS batch, the in vitro dissolution studies showed (fig. 5) around $82.59 \%$ in 5 min and $98.02 \%$ in 45 min. Plain drug revealed very less release of around $35.51 \%$ after $120 \mathrm{~min}$ in 0.1 $\mathrm{N}$ HCL. Through in vitro. dissolution studies, it was shown that the S-SMEDShad improved in the rate of drug release than that of pure drug.

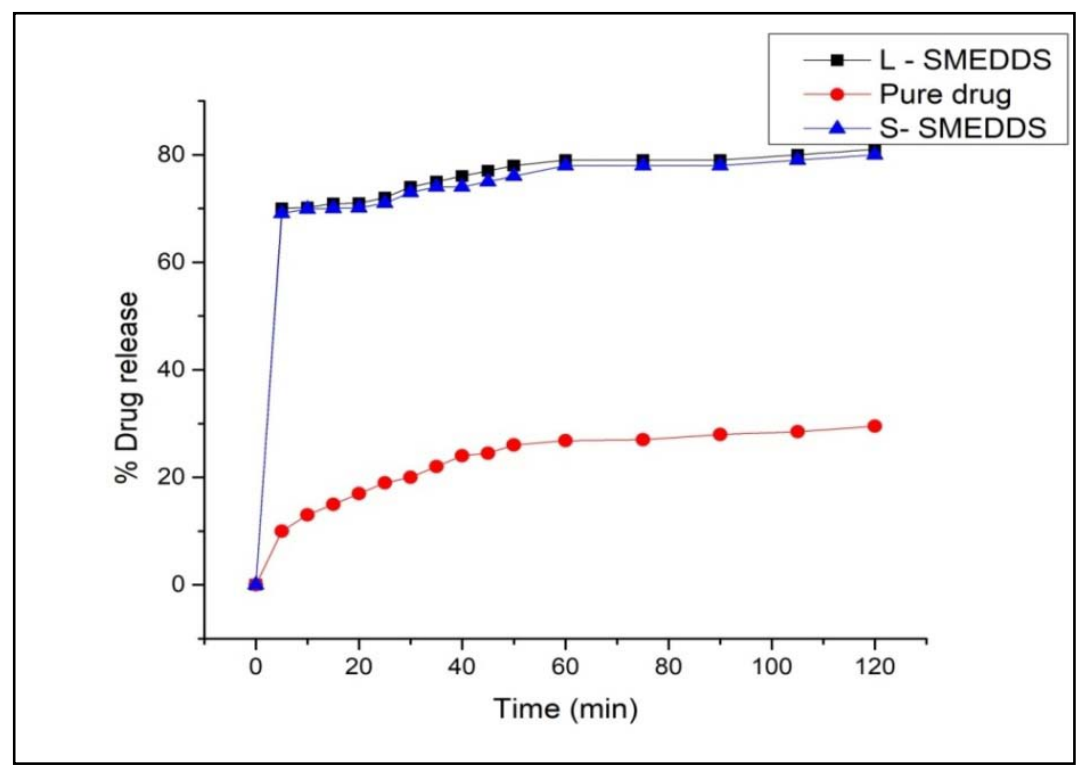


Fig. 5: In vitro dissolution study of PX, S-SMEDS, and L-SMEDS

\section{CONCLUSION}

From the above experiment, the optimized combination of the liquid PX-SMEDS was oil phase (Oleic acid) 23\%, surfactant (Cremophor RH-40) 61\%, co-surfactant (PEG 400) 16\% upon the result obtained by self-emulsifying assess test, solubility test, ternary phase diagrams test and droplet size analysis. The final drug present in the liquid SMEDS was comprised of $10 \%(\mathrm{w} / \mathrm{w})$ An advantageous adsorbent (microcrystalline cellulose MCC KG.802) was affixed to liquid PX-SMEDS to formulate the dispersible tablets utilizing the wet granulation compression technique PX self-micro emulsifying dispersible tablets has developed the solubility as well as the bioavailability of inadequately soluble drugs like PX, and would immediately disperse it into a tiny solid droplet in a dispersion phase. Thus, the above experiment has contributed to the use of the solid self-micro emulsifying system to increase solubility as well as the dissolution of low water-soluble drugs such as PX in the development of future medicinal experimentation.

\section{FUNDING}

Nil

\section{AUTHORS CONTRIBUTIONS}

All authors have contributed equally.

\section{CONFLICT OF INTERESTS}

The author has no conflict of interest to declared.

\section{REFERENCES}

1. Reinholz J, Landfester K, Mailänder V. The challenges of oral drug delivery via nanocarriers. Drug Delivery 2018;25:1694-705.

2. Bennadi D. Self-medication: a current challenge.J Basic Clin Pharm 2013;5:19-23.

3. Obitte NC, Rohan LC, Adeyeye CM, Parniak MA, Esimone CO. The utility of self-emulsifying oil formulation to improve the poor solubility of the anti HIV drug CSIC. AIDS Res Ther 2013;10:14.

4. Yang M, Gong W, Wang Y, Shan L, Li Y, Gao C. Bioavailability improvement strategies for poorly water-soluble drugs based on the supersaturation mechanism: an update. J Pharm Pharm Sci 2016;19:208-25.

5. Lipinski CA, Lombardo F, Dominy BW, Feeney PJ. Experimental and computational approaches to estimate solubility and permeability in drug discovery and development settings. Adv Drug Delivery Rev 1997;23:3-25.

6. Pouton CW. Formulation of poorly water-soluble drugs for oral administration. physicochemical and physiological issues and the lipid formulation classification system. Euro J Pharm Sci 2006;1:278-87.

7. Chaudhary VB, Patel JK. Cyclodextrin inclusion complex to enhance the solubility of poorly water-soluble drugs: a rev. Int J Pharm Sci Res 2013;4:68

8. Sridhar I, Doshi A, Joshi B, Wankhede V, Doshi J. Solid dispersions: an approach to enhance solubility of poorly water soluble drug. J Sci Inno Res 2013;2:685-94.

9. Krishna KB, Prabhakar C. A review on nanosuspensions in drug delivery. Int J Pharma Bio Sci 2011;2:549-8.

10. DV Gowda, G Parthasarathi, HG Shivakumar. Comparative bioavailability of two marketed piroxicam formulations in healthy human volunteers. Ind Drugs 2003;40:573-6.

11. Singh SK, Verma PR, Razdan B. Development and characterization of a lovastatin-loaded self-microemulsifying drug delivery system. Pharm Dev Tech 2010;15:469-83.

12. Hong JY, Kim JK, Song YK, Park JS, Kim CK. A new selfemulsifying formulation of itraconazole with improved dissolution and oral absorption. J Controlled Release 2006;110:332-8.

13. Gohel MC, Nagori SA. Fabrication and evaluation of hydrogel thickened microemulsion of ibuprofen for topical delivery. Ind J Pharm Educ Res 2010;44:189-96.
14. Kuentz M. Lipid-based formulations for oral delivery of lipophilic drugs. Drug Dis Today: Tech 2012;9:97-104.

15. Li S, Madan P, Lin S. Effect of ionization of drug on drug solubilization in SMEDS prepared using capmul MCM and caprylic acid. Asian J Pharm Sci 2017;12:73-82.

16. Nazzal S, Smalyukh II, Lavrentovich OD, Khan MA. Preparation and in vitro characterization of a eutectic-based semisolid selfnano emulsified drug delivery system (SNEDDS) of ubiquinone: mechanism and progress of emulsion formation. Intern J Pharm 2002;235:247-65.

17. Wang YP, Gan Y, Zhang XX. Novel gastroretentive sustainedrelease tablet of tacrolimus based on self-microemulsifying mixture: in vitro evaluation and in vivo bioavailability test. Acta Pharmacol Sin 2011;32:1294.

18. Gupta S, Kesarla R, Omri A. Formulation strategies to improve the bioavailability of poorly absorbed drugs with special emphasis on self-emulsifying systems. ISRN Pharm 2013;26:20-3.

19. DV Gowda, Mohammed S Khan, Afifa Bathool. Formulation and characterization of piroxicam floating microspheres for prolonged gastric retention. Der Pharm Lett 2010;2:217-22.

20. Singh B, Bandopadhyay S, Kapil R, Singh R, Katare OP. Selfemulsifying drug delivery systems (SEDDS): formulation development, characterization, and applications. Critical Rev Thera Drug Carrier Sys 2009;26:5.

21. Bahl D, Bogner RH. Amorphization of indomethacin by cogrinding with Neusilin US2:amorphization kinetics, physical stability and mechanism. Pharm Res 2006;23:2317-25.

22. Suresh S, Gunasekaran S, Srinivasan S. Vibrational spectra (FTIR, FT-Raman), frontier molecular orbital, first hyperpolarizability, NBO analysis and thermodynamics properties of piroxicam by HF and DFT methods. Spect Acta Part A: Mole Biomol Spec 2015;138:447-59.

23. Dhage MA, Chhabra GS, Banerjee SK. Development and validation of UV-spectrophotometric method for PX in bulk and pharmaceutical formulation. J Chem Pharm Res 2011;3:765-9.

24. Kallakunta VR, Bandari S, Jukanti R, Veerareddy PR. Oral selfemulsifying powder of lercanidipine hydrochloride: formulation and evaluation. Pow Tech 2012;221:375-82.

25. Date AA, Nagarsenker MS. Design and evaluation of self-nano emulsifying drug delivery systems (SNEDDS) for cefpodoxime proxetil. Int J Pharm 2007;329:166-72.

26. Czajkowska Kosnik A, Szekalska M, Amelian A, Szymanska E, Winnicka K. Development and evaluation of liquid and solid self-emulsifying drug delivery systems for atorvastatin. Molecules 2015;20:21010-22.

27. Kumar RS. Ghosh, design, optimisation and evaluation of piroxicam fast dissolving tablets employing starch tartrate-a new superdisintegrant. Int J Appl Pharm 2019;11:89-97.

28. Bachhav AA, Ahire SA, Jadhav AG. Preformulation study of piroxicam. Inter J Pharm Sci Res 2019;10:811-8.

29. Nazzal S, Smalyukh I, Lavrentovich OD, Khan MA. Preparation and in-vitro characterization of aeutectic based semisolid selfnanoemulsified drug delivery system (SNEDDS) of ubiquinone: mechanism and progress of emulsion formation. Int J Pharm 2002;235:247-65.

30. Swenson ES, Milisen WB, Curatolo W. Ntestinal permeability enhancement: efficacy, acute local toxicity and reversibility. Pharm Res 1994;11:1132-42.

31. Zeng L, Xin X, Zhang Y. Development and characterization of promising cremophor EL-stabilized $\mathrm{o} / \mathrm{w}$ nanoemulsions containing short-chain alcohols as a cosurfactant. RSC Advances 2017;7:19815-27.

32. Sahoo SK, Suresh P, Acharya U. Design and development of selfmicroemulsifying drug delivery systems (smedds) of telmisartan for enhancement of in vitro dissolution and oral bioavailability in rabbit. Int J Appl Pharm 2018;10:117-26.

33. Nasr A, Gardouh A, Ghonaim H. Abdelghany and Ghorab, Effect of oils, surfactants and cosurfactants on phase behavior and physicochemical properties of self-nanoemulsifying drug delivery system (snedds) for irbesartan and olmesartan. Int J Appl Pharm 2016;8:13-24. 
34. Son HY, Chae BR, Choi JY, Shin DJ, Goo YT, Lee ES, et al. Optimization of self-microemulsifying drug delivery system for phospholipid complex of telmisartan using D-optimal mixture design. PloS one 2018;13:1254. 